\title{
The broad-band spectrum of Cyg X-2 with INTEGRAL (Research Note)
}

\author{
G. Lavagetto ${ }^{1}$, T. Di Salvo ${ }^{1}$, M. Falanga ${ }^{2}$, R. Iaria ${ }^{1}$, N. R. Robba ${ }^{1}$, L. Burderi ${ }^{3}$, W. H. G. Lewin ${ }^{4}$, M. Méndez ${ }^{5}$, \\ L. Stella ${ }^{6}$, and M. van der Klis ${ }^{7}$
}

1 Dipartimento di Scienze Fisiche ed Astronomiche, Università di Palermo, via Archirafi 36, 90123 Palermo (PA), Italy e-mail: lavaget@fisica.unipa.it

2 CEA Saclay, DSM/DAPNIA/Service d'Astrophysique (CNRS FRE 2591), 91191 Gif-sur-Yvette, France

3 Università degli Studi di Cagliari, Dipartimento di Fisica, SP Monserrato-Sestu, KM 0.7, 09042 Monserrato, Italy

${ }^{4}$ Center for Space Research, Massachusetts Institute of Technology, 77 Massachusetts Avenue, Cambridge, MA 02139, USA

5 SRON, National Institute for Space Research, 3584 CA, Utrecht, The Netherlands

${ }^{6}$ Osservatorio Astronomico di Roma, via Frascati 33, 00040 Monteporzio Catone, Roma, Italy

7 Astronomical Institute "Anton Pannekoek", University of Amsterdam, and Center for High Energy Astrophysics, Kruislaan 403, 1098 SJ, Amsterdam, The Netherlands

Received 4 April 2005 / Accepted 14 September 2005

\section{ABSTRACT}

We study the broad band (3-100 keV) spectrum of Cygnus X-2 with INTEGRAL. We find that the spectrum is well fitted by a Comptonized component with a seed-photons temperature of $\sim 1 \mathrm{keV}$, an electron temperature of $\sim 3 \mathrm{keV}$ and an optical depth $\tau \sim 8$. Assuming spherical geometry, the radius of the seed-photons emitting region is $\sim 17 \mathrm{~km}$. The source shows no hard X-ray emission; it was detected only at a $3 \sigma$ level above $40 \mathrm{keV}$. We also analyzed public ISGRI data of Cyg X-2 to investigate the presence of a hard X-ray component. We report the possible presence of hard X-ray emission in one data set.

Key words. accretion, accretion disks - binaries: close - stars: individual: Cygnus X-2 - stars: neutron

\section{Introduction}

Cyg X-2 is one of the six Galactic Low Mass X-ray Binaries (hereafter LMXBs) that are classified as Z-sources. This classification relies upon a combination of the track (that resembles a Z) traced on an X-ray color-color diagram (CCD) and of the correlated timing properties of these sources (Hasinger \& van der Klis 1989).

Several studies have been carried out on Cyg X-2: type-I $\mathrm{X}$-ray bursts have been observed in its X-ray lightcurve (Smale 1998), confirming that the primary in the system is a neutron star (NS); its distance has been estimated to be $7.2 \pm 1.1 \mathrm{kpc}$ from optical observations (Orosz \& Kuulkers 1999), consistent with previous determinations from radio observations (Hjellming et al. 1990) but not with measurements obtained from X-ray bursts (11.6 kpc, Smale 1998). The binary system has an orbital period of $9.8444 \mathrm{~d}$, as can be deduced from the optical behavior of the companion, V1341 Cyg (Casares et al. 1998). The masses of the two stars are $1.78 \pm 0.23 M_{\odot}$ and $0.60 \pm 0.13 M_{\odot}$ for the primary and the companion, respectively (Orosz \& Kuulkers 1999).
The X-ray spectrum of the source has been studied several times over the years. It has been fitted both with the so-called Western model - a blackbody plus a Comptonized component (Hasinger et al. 1990; Smale et al. 1993), and with the socalled Eastern model - a multi-temperature blackbody together with a Comptonized blackbody (Hasinger et al. 1990; Hoshi \& Mitsuda 1991; Hirano et al. 1995). More recently, the broad band spectrum of Cyg X-2 has been studied with BeppoSAX (Frontera et al. 1999; Di Salvo et al. 2002; Piraino et al. 2002).

Di Salvo et al. (2002) fitted the broad band spectrum of Cyg X-2 obtained with BeppoSAX using a two component continuum model, consisting of a disk blackbody and a Comptonized component. A broad emission line at $\sim 1 \mathrm{keV}$ and an emission line at $\sim 6.7 \mathrm{keV}$ (most probably coming from highly ionized iron) proved necessary for a good fit. In two of the intervals selected on the $\mathrm{CCD}$, the continuum spectrum of Cyg X-2 could not be fit by the usual two-component model, and a third component was needed to fit the high energy part of the spectra. This hard X-ray emission fits a power-law with photon index $\sim 2$.

A similar "hard X-ray tail" has been found in other observations, but with an index $\Gamma \sim 3$ (Piraino et al. 2002). 
A hard X-ray emission has been detected in other Z-sources: GX 5-1 (Asai et al. 1994), GX 17+2 (Di Salvo et al. 2000), GX 349+2 (Di Salvo et al. 2001), Sco X-1 (D’Amico et al. 2001a), and in the non-flaring state of the anomalous Z-source Cir X-1 (Iaria et al. 2001). The physical mechanism producing this high-energy emission is yet to be understood. Some evidence of the correlation of the hard tail with the position of the source in the X-ray CCD has been found for GX 5-1, GX 17+2 and Cyg X-2. Such a correlation may not be present in observations of Sco X-1 (D'Amico et al. 2001a) and of GX 349+2 (see Iaria et al. 2004). D'Amico (2001b) proposed that the presence of a high-energy non-thermal emission could be linked to the $20-50 \mathrm{keV}$ luminosity of the Comptonized component of the spectrum: they made the hypothesis that there is a threshold luminosity $\left(\sim 4 \times 10^{36} \mathrm{erg} \mathrm{s}^{-1}\right)$ of the Comptonized component above which the non-thermal hard X-ray emission is produced.

In analyzing the first INTEGRAL pointings to Cyg X-2, Natalucci et al. (2003) found the source in the ISGRI images in the $20-40 \mathrm{keV}$ energy band, while it was not detected above $40 \mathrm{keV}$. We report here on the broad band (3-100 keV) spectral analysis of an INTEGRAL AO1 observation of Cyg X-2 and of all the publicly available ISGRI observations of the source.

\section{Observations}

The AO1 observation was performed between May 2 and May 3, 2003 (52 761.32-52762.43 MJD), during satellite revolution 67. The high-energy coded mask imager IBIS/ISGRI (Ubertini et al. 2003; Lebrun et al. 2003) was used to observe Cyg X-2 for a total exposure time of $66.8 \mathrm{ks}$. Cyg X-2 was in the JEM-X (Lund et al. 2003) field of view during four science windows, for a total exposure of $7.2 \mathrm{ks}$. Data were extracted for all pointings with a source position offset $\leq 12^{\circ}$ in ISGRI and $\leq 3.5^{\circ}$ in JEM-X.

Publicly available data from the satellite pointings towards the Cygnus region during the Performance Verification Phase (PVP) have been previously analyzed (Natalucci et al. 2003). Along with these data, 10 more observations have been carried out with the high-energy coded mask imager and have become publicly available. The publicly available observations used in this paper are listed in Table 1.

Data reduction for both instruments was performed using the standard Offline Science Analysis (OSA) version 4.2 distributed by the INTEGRAL Science Data Center (Courvoisier et al. 2003). The algorithms used in the analysis are described in Goldwurm et al. (2003). For the spectral analysis we used data corresponding to the $3-20 \mathrm{keV}$ energy range for JEM-X and to the $20-100 \mathrm{keV}$ energy range for ISGRI.

\section{Results}

\subsection{The AO1 observation}

Figure 1 shows the ISGRI map of the Cyg X-2 region in the $20-40 \mathrm{keV}$ energy range during the AO1 observation. Single pointings were deconvolved and analyzed separately, and then combined in mosaic images. The source is clearly detected at
Table 1. Summary of all ISGRI observations of Cyg X-2 used in this paper. Start and stop times are in IJD (IJD = MJD - 51 544). The criterion used to detect the hard X-ray emission is discussed in Sect. 3.2. Here we report the power-law flux in the energy range $30-100 \mathrm{keV}$ in units of $10^{-11} \mathrm{erg} \mathrm{cm}^{-2} \mathrm{~s}^{-1}$. Errors and upper limits are at $90 \%$ confidence.

\begin{tabular}{lllllll}
\hline \hline Obs. & Start & End & Exposure (s) & $\begin{array}{l}\text { Rev } \\
\text { PL } \\
\text { norm. }\end{array}$ & $\begin{array}{l}\text { ASM } \\
\text { rate }\end{array}$ \\
\hline 1 & 1085.63 & 1087.82 & 20737 & 023 & $<9.1$ & $29 \pm 1.5$ \\
2 & 1092.68 & 1093.51 & 9169.59 & 025 & $<9.0$ & $54.9 \pm 0.2$ \\
3 & 1109.35 & 1109.39 & 2209 & 031 & $16.8 \pm 8.4$ & $34.4 \pm 0.5$ \\
4 & 1178.89 & 1178.92 & 3308.26 & 054 & $<12.7$ & $31.0 \pm 0.6$ \\
5 & 1202.11 & 1202.17 & 4992.27 & 062 & $<9.2$ & $32.4 \pm 0.6$ \\
6 & 1226.06 & 1226.12 & 4917.2 & 070 & $<9.1$ & $40.5 \pm 0.3$ \\
7 & 1238.05 & 1238.1 & 4889.13 & 074 & $<9.2$ & $39.4 \pm 0.4$ \\
8 & 1257.39 & 1258.34 & 17113.3 & 080 & $<9.2$ & $41.4 \pm 0.6$ \\
9 & 1261.95 & 1262 & 4913.84 & 082 & $<9.2$ & $38.9 \pm 0.9$ \\
10 & 1291.96 & 1292.96 & 5021.14 & 092 & $<7.8$ & $41.0 \pm 1.2$ \\
11 & 1441.42 & 1441.45 & 3308.99 & 142 & $<7.1$ & $35.2 \pm 0.4$ \\
12 & 1450.43 & 1450.48 & 4923.44 & 145 & $<4.0$ & $31.8 \pm 0.6$ \\
AO1 & 1217.37 & 1218.42 & 66795.96 & 067 & $<4.0$ & $45 \pm 3$ \\
\hline
\end{tabular}

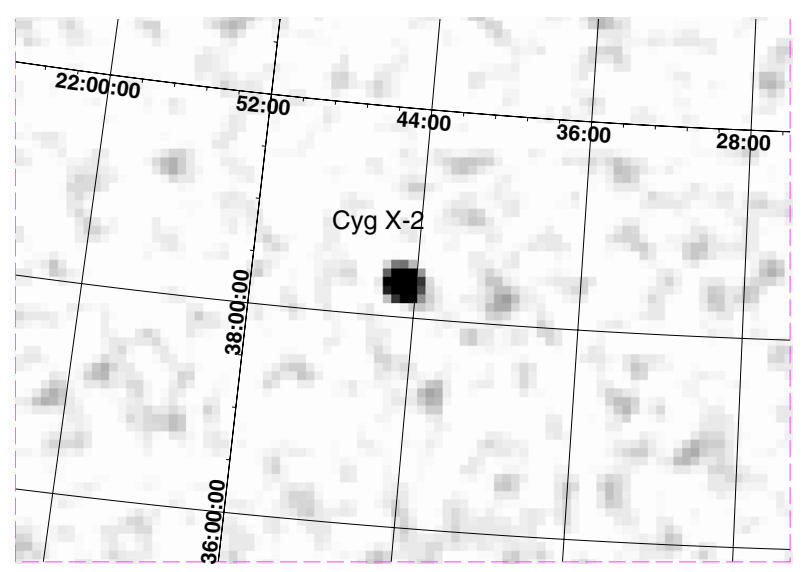

Fig. 1. The 20-40 keV IBIS/ISGRI mosaicked and deconvolved sky image of the AO1 $66.8 \mathrm{ks}$ observation. The image is centered at Cyg X-2 position. The pixel size is $5^{\prime}$.

a significance level of $36 \sigma$. In the energy range $40-80 \mathrm{keV}$, the source can be detected only with a significance of $3 \sigma$. At higher energies Cyg X-2 was not detected at a statistically significant level neither in single exposures nor in the total exposure time. Cyg X-2 was observed in ISGRI images at $\alpha_{\mathrm{J} 2000}=21^{\mathrm{h}} 44^{\mathrm{m}} 40^{\mathrm{s}}$ and $\delta_{\mathrm{J} 2000}=38^{\circ} 19^{\prime} 00^{\prime \prime}$. The source position is consistent, within the source location error at $90 \%$ confidence of $0.8^{\prime}$ (see Gros et al. 2003), with the catalog position (Liu et al. 2001).

We base our spectral investigations mainly on preceding BeppoSAX spectral results (Di Salvo et al. 2002). We find that the broad-band (3-100 keV) spectrum of the AO1 observation is well fitted by an absorbed Comptonization spectrum. Due to the lack of low-energy data we fixed the value of the photoelectric absorption to the value reported by Di Salvo et al. (2002). We will adopt their spectral model in fitting the data. Using the COMPTT (Titarchuk 1994) model in xspec 11.3.1 we were able to fit the data with a reduced chi squared of 1.09 for 144 degrees of freedom (d.o.f.). The non-detection of a low 


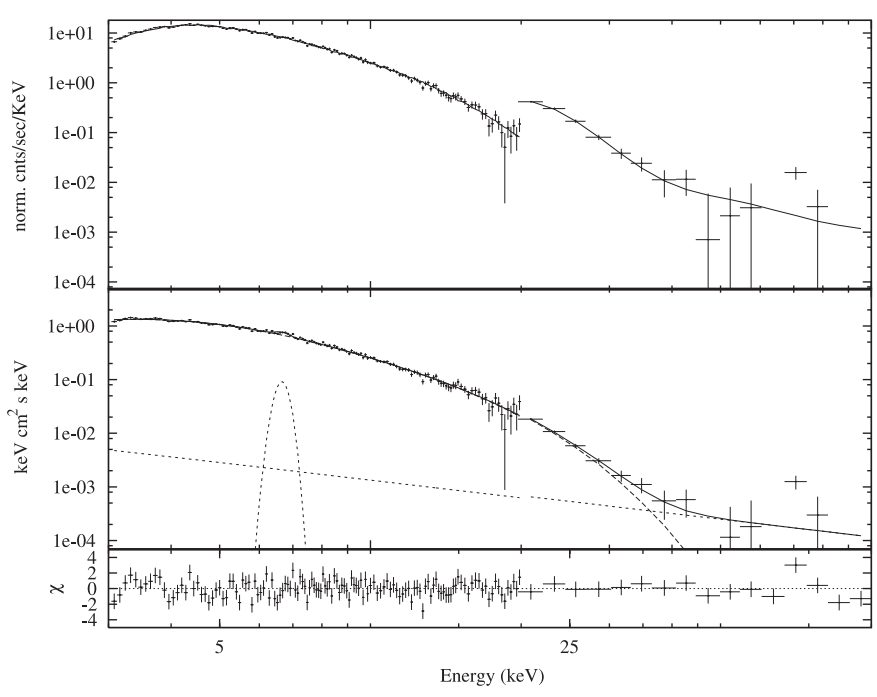

Fig. 2. Folded (top panel) and unfolded (middle panel) spectra of the AO1 Observation of Cyg X-2 (top panel) together with residuals with respect to the best fit model (bottom panel).

energy thermal component in the continuum spectrum is not surprising, since it was used to model mainly the low energy (0.1-3 keV) BeppoSAX spectra. The addition of a power-law component at high energies, or of a Gaussian emission line at $\sim 6.7 \mathrm{keV}$ that were found in BeppoSAX observations are not statistically required. Fixing the energy and the width of the emission line to the best values obtained by Di Salvo et al. (2002), we find an upper limit on the intensity of $13 \times 10^{-3}$ photons $\mathrm{cm}^{-2} \mathrm{~s}^{-1}$ (at $90 \%$ confidence), well compatible with the intensity found in the BeppoSAX observations, which varied between 3 and $7 \times 10^{-3}$ photons $\mathrm{cm}^{-2} \mathrm{~s}^{-1}$. We were able to determine an upper limit on the flux of a power-law component (using the PEGPWRL model inside xspec) by fixing the photon index to the value reported by Di Salvo et al. (2002), 2.09. We find an upper limit (at $90 \%$ confidence) on power law flux between 30 and $100 \mathrm{keV}$ of $4.0 \times 10^{-11} \mathrm{erg} \mathrm{cm}^{-2} \mathrm{~s}^{-1}$. During the BeppoSAX observation analyzed by Di Salvo et al. (2002) in which the hard X-ray emission was detected, the flux in the same energy band was $5.2_{-1.1}^{+7.9} \times 10^{-11} \mathrm{erg} \mathrm{cm}^{-2} \mathrm{~s}^{-11}$. Since the flux in the $3-30 \mathrm{keV}$ energy band in the present observation, $(1.1 \pm 0.3) \times 10^{-8} \mathrm{erg} \mathrm{cm}^{-2} \mathrm{~s}^{-1}$, is comparable with the flux in the BeppoSAX data in the same band, $(1.0 \pm 0.3) \times 10^{-8} \mathrm{erg} \mathrm{cm}^{-2} \mathrm{~s}^{-1}$, we conclude that the spectrum of the INTEGRAL data is softer than the one of the BeppoSAX observations and that no significant hard X-ray emission is present. We added a normalization factor between JEM-X and ISGRI spectra; keeping the ISGRI normalization fixed to 1 we found a JEM-X normalization of 0.986. Detailed results for the best-fit model are reported in Table 2 . The reduced $\chi^{2}$ (d.o.f.) of the best fit model is 1.03(142). The folded and unfolded spectra together with the best-fit model and the respective residuals are shown in Fig. 2.

\footnotetext{
${ }^{1}$ All errors on the fluxes are at $90 \%$ confidence.
}

Table 2. Results of the fit of the INTEGRAL Cyg X-2 spectrum in the 3-100 keV energy band for the AO1 observation, with a Comptonized spectrum modeled by CompTT and with a model consisting of compTT and a power law with the photon index frozen to 2.09. Uncertainties are at $90 \%$ confidence level for a single parameter. Upper limits (also at $90 \%$ confidence) for the normalization of a Gaussian emission line from highly ionized iron and of a hard powerlaw are reported here to make a comparison with results by Di Salvo et al. (2002). $k T_{\mathrm{W}}$ is the temperature of the soft seed photons for the Comptonization, $k T_{\mathrm{e}}$ is the temperature of the scattering electron cloud, while $\tau$ is its optical depth in spherical geometry. $R_{\mathrm{W}}$ is the radius of the seed photons emitting region, assuming a source distance of $7.2 \mathrm{kpc}$. The power-law flux is in units of $10^{-11} \mathrm{erg} \mathrm{cm}^{-2} \mathrm{~s}^{-1}$ in the $30-100 \mathrm{keV}$ energy range. The emission line intensity $I$ is in units of photons $\mathrm{cm}^{-2} \mathrm{~s}^{-1}$. The total unabsorbed flux, in units of $10^{-8} \mathrm{erg} \mathrm{cm}^{-2} \mathrm{~s}^{-1}$, refers to the $3-100 \mathrm{keV}$ energy range.

\begin{tabular}{lll}
\hline \hline & CompTT & $\begin{array}{l}\text { CompTT }+ \\
\text { power-law }\end{array}$ \\
\hline$N_{\mathrm{H}}\left(\times 10^{22} \mathrm{~cm}^{-2}\right)$ & 0.2 (frozen) & 0.2 (frozen) \\
$k T_{\mathrm{W}}(\mathrm{keV})$ & $0.99 \pm 0.03$ & $0.95 \pm 0.04$ \\
$k T_{\mathrm{e}}(\mathrm{keV})$ & $3.44_{-0.34}^{+0.43}$ & $3.03_{-0.31}^{+0.41}$ \\
$\tau$ & $6.8_{-0.82}^{0.87}$ & $8.0_{-1.1}^{+1.2}$ \\
$R_{\mathrm{W}}$ & $17.1 \pm 1.6$ & $18.3 \pm 2.2$ \\
Pho. Index & - & 2.09 (frozen) \\
$N_{\mathrm{po}}$ & - & $<4.0$ \\
$E_{\mathrm{Fe}}(\mathrm{keV})$ & - & 6.65 (frozen) \\
$\sigma_{\mathrm{Fe}}(\mathrm{keV})$ & - & $0.2($ frozen) \\
$I_{\mathrm{Fe}}$ & - & $<13 \times 10^{-3}$ \\
Flux & 1.05 & 1.13 \\
$\chi^{2}($ d.o.f.) & $1.09(144)$ & $1.03(142)$ \\
\hline
\end{tabular}

\subsection{Looking for hard $X$-ray emission in other INTEGRAL observations of Cyg X-2}

Since no JEM-X data are available during other public ISGRI observations, it is not possible to determine the parameters of the COMPTT component at these times. To test the presence of a hard tail, we fitted the ISGRI data with the model we fitted to the AO1 observation, keeping the seed photons temperature $T_{\mathrm{w}}$ and the optical depth of the Comptonizing cloud $\tau$ frozen to their best-fit values; the only free parameters in the fit are therefore the normalizations of the two components and the electron temperature of the COMPTT component. In observations 3, 4 and 11 (see Table 1) we had to fix the electron temperature as well.

We consider that a hard tail is present in the spectrum when the power-law flux in the $30-100 \mathrm{keV}$ energy range is different from 0 at $90 \%$ confidence. Using this criterion, we find one data set in which the hard tail is detected (see Table 1). In the other 11 data sets, no hard tail is detectable, although in most cases the upper limits are consistent with the flux found by BeppoSAX. The detection (or the lack of it) is reported in Table 1. Our results partially confirm that a hard tail appears from time to time in Cyg X-2 spectra, as reported by other authors (Di Salvo et al. 2002; Piraino et al. 2002). It was proposed that the presence of the hard power-law emission is strongest in the horizontal branch of the X-ray CCD (Di Salvo et al. 2002). 
Unfortunately we have no possibility to obtain information about the position of the source along its $\mathrm{Z}$ track, neither in the public data nor during the AO1 observation (JEM-X data covered only a small fraction of the ISGRI observation). In the analysis of the first INTEGRAL observations of Cyg X-2, Natalucci et al. (2003) suggested that there could be a correlation between the variability of the high-energy spectrum and the long term variability in the soft X-ray lightcurve of Cyg X-2. We analyzed the Cyg X-2 lightcurves obtained with the RXTE All Sky Monitor (ASM) simultaneously with the INTEGRAL observations, seeking for some sort of correlation between the long term soft X-ray variability and the detection of a hard tail. We do not find evidence of a correlation between the presence of the hard tail and the count rate in the ASM (see Table 1).

We tried to obtain a crude estimate of the $20-50 \mathrm{keV}$ luminosity of the Comptonized component from our fitting model in order to verify the hypothesis by D'Amico et al. (2001b). In most observations, the error on the inferred luminosity is of one order of magnitude. In the AO1 observation however the power-law was not detected and the $20-50 \mathrm{keV}$ luminosity of the Comptonized component is $(1.0 \pm 0.2) 10^{36} \mathrm{erg} \mathrm{s}^{-1}$, but in observation 3 , where we detect the hard tail, the luminosity of the Comptonized component is $(0.6 \pm 0.5) \times 10^{36} \mathrm{erg} \mathrm{s}^{-1}$. This does not agree with the hypothesis of D'Amico et al. (2001b).

\section{Conclusions}

We analyzed the INTEGRAL AO1 observation of Cyg X-2. The source was clearly detected in the $20-40 \mathrm{keV}$ (see Fig. 1), but only at a $3 \sigma$ level in the $40-80 \mathrm{keV}$ energy band. The broad band spectrum is well fitted by a Comptonization model alone. No hard tail was significantly detected in the data We also analyzed 12 publicly available observations carried out with ISGRI, and confirmed the detection of a hard tail at $90 \%$ confidence in one case. This is the first direct detection of a hard tail in high-energy imaging data of a Z-source. A longer, continuous observation of Cyg X-2 with both JEM-X and ISGRI would allow to investigate further the nature of the hard X-ray emission and its relationship with other spectral features of the source.
Acknowledgements. We acknowledge financial contribution from contract ASI-INTEGRAL I/R/046/04

\section{References}

Asai, K., Dotani, T., Mitsuda, K., et al. 1994, PASJ, 46, 479

Casares, J., Charles, P. A., \& Kuulkers, E. 1998, ApJ, 493, L39

Courvoisier, T. J.-L., Walter, R., Beckmann, V., et al. 2003, A\&A, 411, L57

D’Amico, F., Heindl, W. A., Rothschild, R. E., \& Gruber, D. E. 2001a, ApJ, 547, L147

D'Amico, F. 2001b, AIP Conf. Proc. 587, Gamma 2001, ed. S. Ritz, N. Gehrels, \& C. R. Shrader (Melville, NY: AIP), 44

Di Salvo, T., Stella, L., Robba, N. R., et al. 2000, ApJ, 544, L119

Di Salvo, T., Robba, N. R., Iaria, R., et al. 2001, ApJ, 554, 49

Di Salvo, T., Farinelli, R., Burderi, L., et al. 2002, A\&A, 386, 535

Frontera, F., dal Fiume, D., Malaguti, G., et al. 1999, Nucl. Phys. B Proc. Suppl., 69, 286

Goldwurm, A., David, P., Foschini, L., et al. 2003, A\&A, 411, L223

Gros, A., Goldwurm, A., Cadolle-Bel, M., et al. 2003, A\&A, 411, L179

Hasinger, G., \& van der Klis, M. 1989, A\&A, 225, 79

Hasinger, G., van der Klis, M., Ebisawa, K., Dotani, T., \& Mitsuda, K. 1990, A\&A, 235, 131

Hirano, A., Kitamoto, S., Yamada, T. T., Mineshige, S., \& Fukue, J. 1995, ApJ, 446, 350

Hjellming, R. M., Han, X. H., Cordova, F. A., \& Hasinger, G. 1990, A\&A, 235, 147

Hoshi, R., \& Mitsuda, K. 1991, PASJ, 43, 485

Iaria, R., Di Salvo, T., Burderi, L., \& Robba, N. R. 2001, ApJ, 561, 321

Iaria, R., Di Salvo, T., Robba, N. R., et al. 2004, ApJ, 600, 358

Lebrun, F., Leray, J.-P., Lavocate, Ph., et al. 2003, A\&A, 411, L141

Liu, Q. Z., van Paradijs, J., \& van den Heuvel, E. P. J. 2001, A\&A, 368,1021

Lund, N., Budtz-Joergensen, C., Westgaard, N. J., et al. 2003, A\&A, 411, L231

Natalucci, L., Del Santo, M., Ubertini, P., et al. 2003, A\&A, 411, L395

Orosz, J. A., \& Kuulkers, E. 1999, MNRAS, 305, 132

Piraino, S., Santangelo, A., \& Kaaret, P. 2002, ApJ, 567, 1011

Smale, A. P., Done, C., Mushotzky, R. F., et al. 1993, ApJ, 410, 796

Smale, A. P. 1998, ApJ, 498, L141

Titarchuk, L. 1994, ApJ, 434, 570

Ubertini, P., Lebrun, F., Di Cocco, G., et al. 2003, A\&A, 411, L131 